\title{
Bilateral symmetry of linear streptomycete chromosomes
}

\author{
Lis Algora-Gallardo', Jana K. Schniete ${ }^{1,2}$, David R. Mark¹, lain S. Hunter ${ }^{1}$ and Paul R. Herron ${ }^{1, *}$
}

\begin{abstract}
Here, we characterize an uncommon set of telomeres from Streptomyces rimosus ATCC 10970, the parental strain of a lineage of one of the earliest-discovered antibiotic producers. Following the closure of its genome sequence, we compared unusual telomeres from this organism with the other five classes of replicon ends found amongst streptomycetes. Closed replicons of streptomycete chromosomes were organized with respect to their phylogeny and physical orientation, which demonstrated that different telomeres were not associated with particular clades and are likely shared amongst different strains by plasmiddriven horizontal gene transfer. Furthermore, we identified a $\sim 50 \mathrm{~kb}$ origin island with conserved synteny that is located at the core of all streptomycete chromosomes and forms an axis around which symmetrical chromosome inversions can take place. Despite this chromosomal bilateral symmetry, a bias in parS sites to the right of oriC is maintained across the family Streptomycetaceae and suggests that the formation of ParB/parS nucleoprotein complexes on the right replichore is a conserved feature in streptomycetes. Consequently, our studies reveal novel features of linear bacterial replicons that, through their manipulation, may lead to improvements in growth and productivity of this important industrial group of bacteria.
\end{abstract}

\section{DATA SUMMARY}

Supplementary data files are available from Figshare: https:// doi.org/10.6084/m9.figshare.16553307 [1]. Sequence data for the Streptomyces rimosus ATCC 10970 chromosome and plasmid are deposited with the National Center for Biotechnology Information (NCBI) under accession numbers NZ CP048261.1 and NZ_CP048262.1, respectively (BioSample SAMN02471950; BioProject PRJNA182749). The location and identities of all putative parS sites across all 20 closed streptomycete genome sequences are provided in Supplementary File S1 (available with the online version of this article and via Figshare).

\section{INTRODUCTION}

Streptomycetes are important industrial and environmental bacteria with large linear chromosomes of up to $12 \mathrm{Mb}$ in size [2]. They are renowned for their extensive metabolic repertoire, producing metabolites used in healthcare and industry [3]. The bacteria undergo a complex life cycle involving germination from dormant spores, vegetative growth, differentiation and sporulation again, whilst production of the useful metabolites is intrinsically linked with the developmental life cycle [4]. With this as background, we undertook an analysis of the genome of Streptomyces rimosus, the commercial producer of oxytetracycline. Accurate prediction of the molecules produced by these organisms is dependent on the presence of a complete genome sequence; in the case of organisms with linear chromosomes, knowledge of the telomeres of individual replicons is essential to delineate the full coding capacity of the genome and also to gain understanding of its basic architecture. Following the closure of the S. rimosus genome sequence, we noticed that the locations of certain genes and sequence motifs required for effective replication and segregation of its genetic material were shared with other streptomycetes. Bidirectional DNA replication in most bacteria begins at a central origin (oriC) and proceeds around the chromosome until the two replication forks meet at the terminus (ter) of the circular molecule [5]. Streptomycetes possess linear chromosomes and plasmids [6] flanked

Received 04 June 2021; Accepted 14 September 2021; Published 15 November 2021

Author affiliations: 'Strathclyde Institute of Pharmacy and Biomedical Sciences, University of Strathclyde, Glasgow G4 ORE, UK; ${ }^{2}$ Department of Biology, Edge Hill University, Ormskirk L39 4QP, UK.

${ }^{*}$ Correspondence: Paul R. Herron, paul.herron@strath.ac.uk

Keywords: bilateral symmetry; linear chromosome; oriC; Streptomyces.

Abbreviations: GLP, giant linear plasmid; LCB, locally collinear block; MLSA, multilocus sequence analysis; NCBI, National Center for Biotechnology

Information; TIR, terminal inverted repeat.

The NCBI accession numbers for the chromosome and plasmid sequences of Streptomyces rimosus ATCC 10970 are NZ_CP048261.1 and NZ_

CP048262.1, respectively (BioSample SAMN02471950; BioProject PRJNA182749).

Data statement: All supporting data, code and protocols have been provided within the article or through supplementary data files. Four supplementary tables, seven supplementary figures, one supplementary information and one supplementary file are available with the online version of this article. $000692 \odot 2021$ The Authors 
by terminal inverted repeats (TIRs) that can be over $600 \mathrm{~kb}$ in size [7] and end in telomeres that can form complex stem-loop structures [8]. Here, we use the term streptomycete to refer to members of the three genera that form the family Streptomycetaceae: Streptomyces, Kitasatospora and Streptacidiphilus [9]. Archetypal chromosome ends (telomeres) are the best characterized termini of streptomycete linear replicons and contain several palindromes with differences in sequence that occur in complementary pairs of stem structures in the terminal $150 \mathrm{bp}$ [8]. These palindromes adopt a clover-leaf structure required for priming by palindrome I in archetypal telomeres [10]. However, there are also non-archetypal telomeres, such as those of SCP1, a giant linear plasmid (GLP) of Streptomyces coelicolor. In addition, a number of other non-archetypal telomeres have been identified, such as those from the chromosomes of Streptomyces griseus 2247, S. griseus 13350 and two linear plasmids, pLR1 and pLR2 [11-13]. Whist all known telomeres contain palindromes, their sequences differ between each class (Fig. 1).

While end-patching of archetypal streptomycete telomeres is well understood and is carried out by the telomeric proteins, Tap and Tpg [10], there is still a gap in our knowledge for most other types of telomeres. tap and tpg are essential for the end-patching of linear streptomycete replicons with archetypal telomeres, as their deletion leads to replicon circularization [14]. Tac and Tpc are required for end-patching of SCP1 telomeres [15], but there is no mechanistic information for end-patching of other non-archetypal streptomycete telomeres.

The main aim of this research was to complete the genome sequence of $S$. rimosus ATCC $10970[16,17]$. Key to this was to determine the telomeric sequences of the two S. rimosus replicons: a chromosome and GLP, first identified by PFGE $[18,19]$. However, when attempting a comparison with other streptomycete genomes, we were only able to identify a small number of streptomycete genome sequences flanked by known telomeres and, thus, truly closed. We then set out to analyse these closed sequences for the location of oriC, parS sites and genes encoding the telomeric proteins (Tap/Tpg) and partitioning proteins (ParA/ParB) [20, 21]. In so doing, we identified shared features of streptomycete linear chromosomes that increase our understanding of how genomic architecture is conserved in this important antibiotic-producing bacterial group.

\section{METHODS}

\section{Genomic DNA extraction, sequencing and genome assembly}

S. rimosus was obtained from Pfizer [16] and propagated on Emerson's agar at $30^{\circ} \mathrm{C}\left(4 \mathrm{~g}\right.$ beef extract $\mathrm{l}^{-1}, 1 \mathrm{~g}$ yeast extract $\mathrm{l}^{-1}, 4 \mathrm{~g}$ peptone $\mathrm{l}^{-1}, 10 \mathrm{~g}$ glucose $\mathrm{l}^{-1}, 2.5 \mathrm{~g} \mathrm{NaCl} \mathrm{l}^{-1}, 20 \mathrm{~g}$ agar $\mathrm{l}^{-1}, \mathrm{pH}$ 7.2) before inoculating Tryptone Soya Broth (Oxoid) [22], incubated at $30^{\circ} \mathrm{C}, 250$ r.p.m. in $250 \mathrm{ml}$ baffled flasks for $36 \mathrm{~h}$ prior to genomic DNA isolation or preparation of PFGE plugs. For the former, biomass was harvested by centrifugation and resuspended in $300 \mu \mathrm{l}$ TE buffer, $\mathrm{pH} 7.5$, with 10

\section{Impact Statement}

After closing the genome sequence of Streptomyces rimosus, one of the earliest-discovered antibioticproducing bacteria, we identified an uncommon set of chromosome and plasmid ends. Unusually amongst bacteria, streptomycetes have linear chromosomes and further analysis of closed streptomycete genomes allowed us to bring together a description of the known telomeres found in this family for the first time. We also identified that different ends were not associated with particular groups and are likely shared amongst different strains by plasmid-driven horizontal gene transfer, potentially responsible for the evolution of the great metabolic diversity displayed by this bacterial group. Furthermore, we identified a genomic island located at the core of streptomycete chromosomes that forms an axis around which symmetrical chromosome inversions can take place. Despite this bilateral symmetry, a bias in pars sites to the right of the replication origin is maintained across the streptomycetes and suggests that the formation of ParB/parS nucleoprotein complexes on the right-hand arm of the chromosome is a conserved feature in streptomycetes. Consequently, our studies reveal novel features of streptomycete replicons that expand our understanding of genomic rearrangements in bacteria that may lead to improvements in the productivity of this important industrial group of bacteria.

mg lysozyme $\mathrm{ml}^{-1}$ and RNase $\left(0.1 \mathrm{mg} \mathrm{ml}^{-1}\right)$ and incubated for $90 \mathrm{~min}$ at $37^{\circ} \mathrm{C}$. A $50 \mu \mathrm{l}$ aliquot of $10 \%$ (w/v) SDS was added and the sample mixed, followed by addition of 85 $\mu \mathrm{l} 5 \mathrm{M} \mathrm{NaCl}$. A total of $400 \mu \mathrm{l}$ phenol/chloroform/isoamyl alcohol (25:24:1) was added, vortexed for $30 \mathrm{~s}$ and centrifuged for $10 \mathrm{~min}$ at 8000 r.p.m. The aqueous layer was added to a new tube and the previous two steps were repeated. A total of $400 \mu \mathrm{l}$ chloroform/isoamyl alcohol (24:1) were added and vortexed for $30 \mathrm{~s}$ and centrifuged for $10 \mathrm{~min}$ at 8000 r.p.m. The aqueous layer was added to a new tube and $0.5 \mathrm{ml}$ isopropanol added. The tube was inverted and incubated for $5 \mathrm{~min}$ at room temperature. DNA was pelleted at 10000 r.p.m., washed with $70 \%(\mathrm{v} / \mathrm{v})$ ethanol, the supernatant removed and the pellet air dried for $30 \mathrm{~min}$ at room temperature. The DNA was resuspended in $10 \mathrm{mM}$ Tris buffer $\mathrm{pH} 7.5$ and sent for sequencing by PacBio at NU-OMICS (https://www.northumbria.ac. uk/business-services/engage-with-us/research/nu-omics/) and by Illumina $2 \times 250$ bp paired-end reads at MicrobesNG (https://microbesng.com/). DNA was quantified using the Qubit dsDNA HS assay. Details of sequence assembly are provided in the Supplementary Information.

\section{Determination of telomere sequences}

Purification of the telomeric sequences was performed following an adaptation of a previously described procedure [23]. A total of $1 \mu \mathrm{g}$ genomic DNA of S. rimosus was digested 


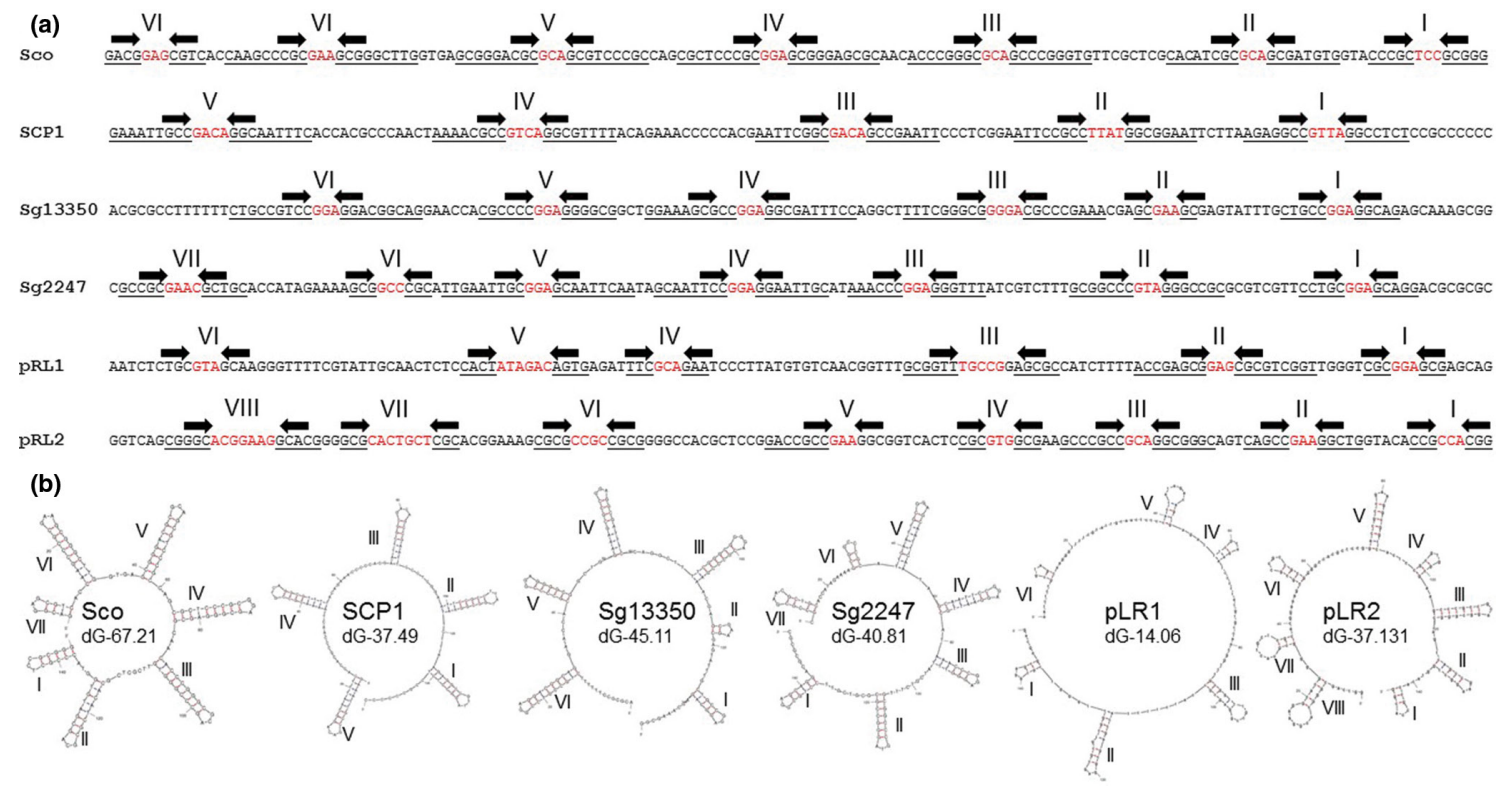

Fig. 1. The six known classes of streptomycete telomeres. (a) Terminal $150 \mathrm{bp}$ of $3^{\prime}$ replicon ends of known streptomycete telomeres showing stem structures (underlined, arrows) and hairpins (red) identified using Mfold (I-VIII). Sco, archetypal end from S. coelicolor chromosome [25]; SCP1, non-archetypal end from S. coelicolor plasmid SCP1 [15]; Sg13350, chromosome end from S. griseus 13350 chromosome [13]; Sg2247, chromosome end from S. rimosus ATCC 10970 chromosome (this work); pRL1, end from Streptomyces sp. 44030 plasmid pRL1 [12]; pRL2, end from Streptomyces sp. 440414 plasmid pRL2 [12]. (b) Mfold projections of the terminal 150 bp of the known classes of streptomycete telomeres showing stem-loop structures (I-VIII) identified using Mfold [28] are displayed, where the revised free energies $(\Delta G)$ were determined using Jacobson-Stockmeyer theory to assign free energies to multi-branch loops. Further details of known streptomycete telomeres are displayed in Table S2 and each individual Mfold representation in Fig. S4.

overnight with the blunt-end restriction enzymes SmaI and PvuII. The products were then purified using the Promega PCR Clean-Up kit and eluted in a final volume of $90 \mu \mathrm{l}$ with $\mathrm{dH}_{2} \mathrm{O}$. A total of $10 \mu \mathrm{l} 1 \mathrm{M} \mathrm{NaOH}$ was added and the mixture was incubated for $1 \mathrm{~h}$ at $37^{\circ} \mathrm{C}$. The samples were neutralized using $2 \mathrm{M} \mathrm{HCl}$ and $1 \mathrm{M}$ Tris ( $\mathrm{pH} 8$ ) was added to a final concentration of $0.1 \mathrm{M}$. A $20 \times$ SSC solution was then added to final concentration of $2 \times$ and the samples were incubated at $68^{\circ} \mathrm{C}$ for $1 \mathrm{~h}$. The samples were purified once again with the Promega PCR Clean-Up kit and ligated overnight using Promega T4 DNA ligase. Inverted PCR analyses were performed using the primers listed in Table S1, and the amplified products were purified and sequenced by Eurofins Genomics.

\section{PFGE}

PFGE analyses were performed using the Bio-Rad CHEF-DR II PFGE system. Agarose plugs containing DNA were prepared from TSB grown liquid cultures of S. rimosus using established procedures [22], with the following amendments to reduce DNA degradation [24]. HEPES was substituted for Tris in buffers and mycelium was washed in HES buffer (25 mM HEPES-NaOH, $25 \mathrm{mM}$ EDTA, $0.3 \mathrm{M}$ sucrose, $\mathrm{pH} 8$ ) and digested in $1 \mathrm{mg}$ lysozyme $\mathrm{ml}^{-1}$ in HES buffer. After lysis, plugs were washed three times with NDS (1\% $N$-laurylsarcosine, $0.5 \mathrm{M}$ EDTA, $10 \mathrm{mM}$ glycine, $\mathrm{pH}$ 9.5). The plugs were incubated overnight with NDS containing $1 \mathrm{mg}$ proteinase $\mathrm{K} \mathrm{ml}^{-1}$ and $1 \mathrm{mM} \mathrm{CaCl}$. The next day, the plugs were washed in $\mathrm{HE}$ buffer (10 mM HEPES-NaOH, 1 mM EDTA, pH 8). After $15 \mathrm{~min}$ at room temperature, the liquid was replaced with fresh buffer and $1 \mu \mathrm{l} \mathrm{BSA}\left(10 \mu \mathrm{g} \mathrm{ml}^{-1}\right)$, and $50 \mathrm{U}$ AseI or DraI added. The mix was incubated overnight at $37^{\circ} \mathrm{C}$. In the case of AseI digestion, a second sample of $10 \mathrm{U}$ enzyme was added after $2 \mathrm{~h}$. For electrophoresis, HEPES buffer (16 mM HEPES$\mathrm{NaOH}, 16 \mathrm{mM}$ sodium acetate, $0.8 \mathrm{mM}$ EDTA, pH7.5) was used and the voltage set at $4 \mathrm{~V} \mathrm{~cm}^{-1}$, with an initial switch time of $70 \mathrm{~s}$ and a final switch time of $130 \mathrm{~s}$. Electrophoresis was performed for $24 \mathrm{~h}$. The gel was then stained for $30 \mathrm{~min}$ in 1 $\mu \mathrm{g}$ ethidium bromide $\mathrm{ml}^{-1}$ solution and de-stained with $\mathrm{dH}_{2} \mathrm{O}$ for an hour. The gel was imaged with a UV trans-illuminator.

\section{Telomere identification and analysis}

In order to identify streptomycete telomeres, we used the terminal 36 and $150 \mathrm{bp}$ of the six classes of telomeres to search the National Center for Biotechnology Information (NCBI) database using BLASTN. The two sizes of query sequences were taken from: the S. coelicolor chromosome end (Sco) [25]; the end of S. coelicolor plasmid SCP1 (SCP1) [15]; the chromosome end of S. griseus 13350 (Sg13350) [13]; the chromosome end of S. rimosus ATCC 10970 (Sg2247) (this work); the end of Streptomyces sp. 44030 plasmid pRL1 (pRL1) [12]; the end of Streptomyces sp. 440414 plasmid pRL2 (pRL2) [12]. The query sequences are described in Fig. 1. Only those significant hits from complete whole-genome sequencing projects that 
were either located at the end of a replicon or where sequences were physically recovered and sequenced were designated as telomeres.

A ClustalW alignment of 13 Sg2247 independent telomeres was used to map telomere evolutionary history and was inferred using the maximum-likelihood method and the Tamura three-parameter model [26]. The tree with the highest log likelihood $(-1238.57)$ was used. Initial tree(s) for the heuristic search were obtained automatically by applying Neighbor-Join and BioNJ algorithms to a matrix of pairwise distances estimated using the maximum composite likelihood (MCL) approach, and then selecting the topology with superior log likelihood value. A discrete gamma distribution was used to model evolutionary rate differences among sites [five categories $(+\mathrm{G}$, parameter $=0.6336)$ ]. Trees were drawn to scale, with branch lengths measured by the number of substitutions per site. This analysis involved 13 nucleotide sequences and there were 163 positions in total in the final dataset. Evolutionary analyses were conducted in MEGA X [27].

Stem-loop structures identified using Mfold [28] are displayed, where the revised free energies $\left(\Delta \mathrm{G}, \mathrm{kJ} \mathrm{mol}^{-1}\right)$ were determined using Jacobson-Stockmeyer theory to assign free energies to multi-branch loops. Projections were calculated using default conditions except folding temperature was set at $30{ }^{\circ} \mathrm{C}, \mathrm{Na}^{+}$concentration $0.05 \mathrm{M}$ and maximum distance between paired bases was set at 20 [10]. Closed chromosome sequences from the genus Streptomyces, in conjunction with the closed sequence of Kitasatospora setae KM-6054 as an outgroup, were used to carry out multilocus sequence analysis (MLSA) to produce a high-resolution species tree using AutoMLST after a concatenated alignment [29]. The average nucleotide identities between 20 closed streptomycete genomes were calculated using the OrthoANI tool [30] and plotted with the heat map function in $\mathrm{R}$ (ver. 4.0).

Closed streptomycete chromosomes were subjected to a progressive alignment in Mauve [31] using default settings and dot plots generated by Nucmer (Galaxy version 4.0.0beta2 +galaxy0) [32] with default settings, except that the filter setting was turned on so only delta alignments which represent the 'best' hit to any particular location on either sequence were displayed. Maps showing synteny or origin islands were created using clinker and clustermap.js [33].

The locations of predicted parS sites were determined by searching the 20 closed genomes using the consensus matrix for bacterial parS sites [34]. This was done by performing a ClustalW alignment of the 1030 predicted parS sited previously identified [34]. A consensus matrix was then constructed in WebLogo 3 (Galaxy version 4.0.0beta2 + galaxy0) [35] and used to interrogate closed streptomycete genomes using matrix scan in the Regulatory Sequence Analysis Tools suite (RSAT) (http://embnet.ccg.unam.mx/rsat//matrix-scanquick_form.cgi) [36]. For this analysis the threshold weight score was set at $>15$ [34].

\section{RESULTS AND DISCUSSION \\ Closed genome of S. rimosus ATCC 10970 consists of two linear replicons}

S. rimosus is one of the most sequenced streptomycetes with 38 records listed in the NCBI database at time of writing (assembly level: complete, 2; chromosome, 1; scaffold, 2; contig, 33), although none contain recognizable streptomycete telomeres. Analysis of $32 \mathrm{~S}$. rimosus genome sequences shows that members of this species encode between 35 and 71 specialized metabolite biosynthetic gene clusters [37]. Many streptomycetes contain specialised metabolite biosynthetic gene clusters in the arms of linear chromosomes [38], whose annotation is likely complicated by the presence of multiple contigs and TIRs. Consequently, we used a combination of PacBio and Illumina sequencing, in conjunction with the physical recovery and sequencing of the chromosome and plasmid ends, to generate a fully closed sequence of $S$. rimosus. The genome assembly details are described in the Supplementary Information.

The genome of S. rimosus ATCC 10970 consists of a linear chromosome and plasmid, SRP1 (Fig. S1a), which is consistent with earlier physical studies $[18,19]$. The chromosome is $9351267 \mathrm{bp}$ in size with 11386 bp TIRs. The GLP, SRP1, is 292624 bp in size with TIRs of 288 bp. Together these two replicons encode 8292 coding sequences, 7 rRNA operons, 68 tRNAs and 3 non-coding RNAs. The S. rimosus ATCC 10970 genome is listed in the NCBI database under accession numbers NZ_CP048261.1 (chromosome) and NZ_CP048262.1 (plasmid). The size of the GLP, SRP1, was verified by PFGE and the assemblies of both replicons were verified by DraI and AseI restriction digestion followed by PFGE (Fig. S1b). The restriction patterns observed agreed with the in silico predictions of their size and corroborates our assembly. PFGE patterns from S. rimosus R7 (R7 and G7 are synonyms for ATCC 10970) were similar to those from S. rimosus R6 [19] except that an additional fragment was located at one chromosome end of R7. This suggests R6 and R7 share a common ancestor and might account for the different telomeres found in S. rimosus R6 and R7 [16] and reported here.

\section{Replicons of S. rimosus ATCC 10970 are flanked by rare telomeres}

To close the genome sequence of S. rimosus ATCC 10970, we employed a self-ligation PCR-sequencing method [23] to recover the telomeres of the chromosome and SRP1. Assembly of Illumina and PacBio reads produced two contigs corresponding to these replicons (see the Supplementary Information) and allowed us to identify SmaI and PvuII as restriction enzymes that digested close to the ends of both molecules. When the $S$. rimosus genomic DNA was digested with SmaI and PvuII and the fragments were self-ligated, circular molecules were generated that corresponded to the ends of both replicons (Fig. S2). Using the primers listed in Table S1, the telomeres were recovered as PCR products and sequenced. These telomere sequences were combined with 


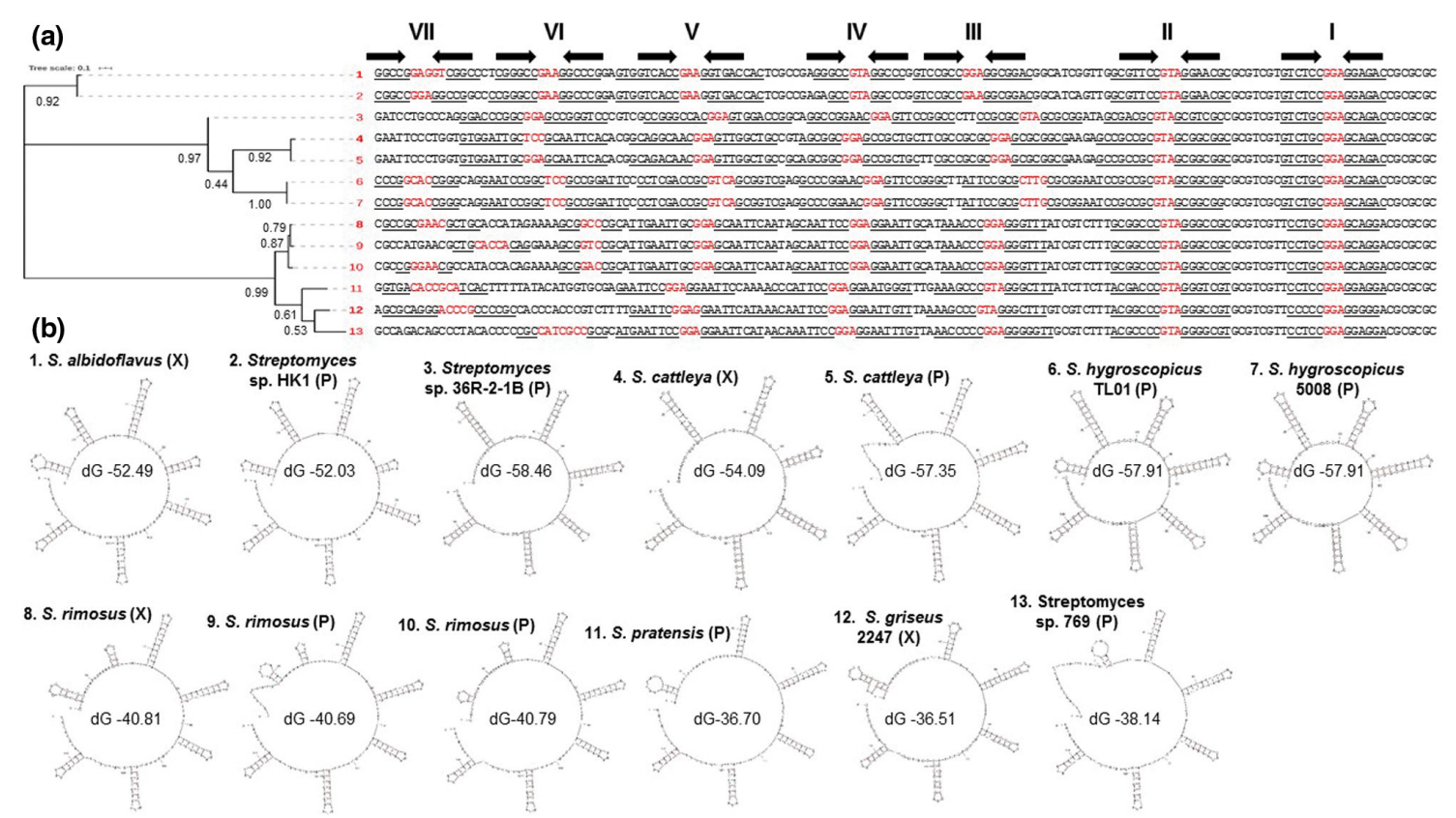

Fig. 2. Evolutionary relationships of Sg2247-type telomeres showing stem-loop structures. (a) Phylogenetic tree of $3^{\prime}$ replicon end sequences with similarity to the telomeres of S. rimosus ATCC 10970 (Sg2247-type telomeres) [11]. The proportion of trees in which the associated taxa clustered together is shown next to the branches. The tree is drawn to scale, with branch lengths measured in the number of substitutions per site. 1, S. albidoflavus J1074 chromosome, accession number NC_020990.1; 2, Streptomyces sp. HK1 plasmid pSHK1, EU372836.1; 3, Streptomyces sp. 36R-2-1B plasmid pYY8L, GU080325.1; 4, 'S. cattleya' NRRL 8057 chromosome, FQ859185.1; 5, 'S. cattleya' NRRL 8057 plasmid pSCAT, FQ859184.1; 6, S. hygroscopicus subsp. jinggangensis TL01 plasmid pSHJGH1 right-hand end, NC_020894.1; 7, S. hygroscopicus subsp. jinggangensis 5008 plasmid pSHJG1 right-hand end, NC_017766.1; 8, S. rimosus ATCC 10970 chromosome, CP048261.1; 9, S. rimosus ATCC 10970 plasmid SRP1 left-hand end, CP048261.2; 10, S. rimosus ATCC 10970 plasmid SRP1 right-hand end, CP048261.2; 11, Streptomyces pratensis ATCC 33331 plasmid pSFLA01, CP002476.1; 12, S. griseus 2247 chromosome; 13. Streptomyces sp. 769 plasmid pSGZL, CP003988.1. (b) The terminal 150 bp of 3' replicon ends of Sg2247-type telomeres showing stem structures and hairpins identified using Mfold [28] are displayed. Revised free energies ( $\Delta \mathrm{G}, \mathrm{kJ}_{\mathrm{mol}}{ }^{-1}$ ) were determined using Jacobson-Stockmeyer theory to assign free energies to multi-branch loops. Those telomeres located on chromosomal $(X)$ and plasmid (P) replicons are designated in parentheses. Further details of known Sg2247 telomeres are displayed in Table S2 and each individual Mfold representation in Fig S3.

the draft sequence of both replicons to close the sequences. Here, we define a closed streptomycete sequence to include the entire sequence of all replicons; many sequences listed as complete by the NCBI do not contain recognizable telomeres. The chromosomal telomeres had identical sequences, whilst the left- and right-hand telomeres of SRP1 had a high degree of similarity (Fig. 2) to each other and to a group of telomeres first found in S. griseus 2247 [11] that are distinct from archetypal and other non-archetypal telomeres. Although the telomeric sequence from this strain has not been submitted to the NCBI, we named this class of telomeres as Sg2247. An archetypal telomeric sequence exists in the NCBI database from the telomere of S. rimosus R6 [8] (accession number AY043328.1) and suggests that telomeric heterogeneity exists within strains of this species. S. rimosus ATCC 10970 and S. rimosus $\mathrm{R} 6$ are independent isolates: the former is the original soil isolate [39] and the latter is a soil isolate from Zagreb, Croatia [16].

We next set out to determine the incidence of Sg2247-type telomeres across the streptomycetes. We used the terminal 150 bp of the S. rimosus chromosome telomere to search the
NCBI database using BLASTN. This generated over 50 hits, with a query coverage and identity of at least 19 and $76 \%$, respectively. To exclude pseudo-telomeres $[15,40]$ and detect true telomeric sequences, we only included sequences that were found at the end of a replicon from complete genomes or where the telomere sequence had been physically isolated and sequenced. Eleven replicons contained telomeres similar to those of S. rimosus (Sg2247-type); two were at both chromosome ends (S. rimosus ATCC 10970 and Streptomyces albidoflavus J1074) [41], whilst 'Streptomyces cattleya' NRRL8057 [42] possesses a Sg2247 telomere at one end and no recognizable telomere at the other. In addition, Sg2247-type telomeres were found at both termini of three plasmids and at one terminus of a further four plasmids. Interestingly, two plasmids (pSHJG1 from Streptomyces hygroscopicus subsp. jinggangensis 5008 [43] and pSHJGH1 from S. hygroscopicus subsp. jinggangensis TL01) contained a Sg2247-type telomere at one terminus and an archetypal telomere at the other.

A ClustalW alignment of 13 Sg2247 telomeres was used to determine the evolutionary relationships within this class of telomeres (Fig. 2a). Sg2247-type telomeres form similar 
stem-loop structures to those found in archetypal and non-archetypal telomeres [15], with up to eight stem-loop structures predicted within the terminal $150 \mathrm{bp}$ and highest similarity at their $3^{\prime}$ ends where terminal stem-loop structures are highly conserved (Fig. 2, individual Mfold projections are shown in Fig. S3). The most common sequences found at the hairpins were GGA, GAA and GTA, like that found at the hairpins of archetypal telomeres [15]. Revised free energies $(\Delta \mathrm{G})$ of the stem-loop structures predicted by Mfold [28] varied between -36.51 and $-58.46 \mathrm{~kJ} \mathrm{~mol}^{-1}$. Sg2247 telomeres fell into two groups that reflected their phylogenetic relationship (Fig. $2 \mathrm{~b}$ ): those with a relatively low $\Delta \mathrm{G}$ (strains $1-7)$ and those with a significantly higher $\Delta \mathrm{G}$ [strains $8-13$, according to a Student's $t$-test $\left.\left(P<6.47 \times 10^{-7}\right)\right]$. Strains with the lowest $\Delta \mathrm{G}$ (strains 1-7) contained longer hairpins distal to the $3^{\prime}$ replicon end, although it is unclear whether this has any functional significance.

\section{Six classes of telomeres are found in the linear replicons of streptomycetes}

Archetypal telomeres are found in many streptomycete replicons, such as the chromosome end of S. coelicolor M145 [25]. Non-archetypal telomeres were first identified in SCP1, the GLP of S. coelicolor A3(2) [15], and another class of telomeres was identified in the S. griseus 13350 chromosome [13], here termed Sg13350-type. Finally, two types of telomeres were found at the ends of two linear plasmids, pRL1 and pRL2 from streptomycete soil isolates [12]. Using the same approach with which we identified Sg2247-type telomeres, we also compiled a list of telomeres from all six classes that were found at the end of closed replicons or had been recovered physically and sequenced by Sanger sequencing [23]. The predicted streptomycete telomeres of all six classes are listed in Table S2. Archetypal telomeres are the most frequent and are found in both chromosomes and linear plasmids, whilst three SCP1type telomeres were identified (SCP1) [15], pSCO2 [7] and pFRL3 (Chen and others, unpublished data, 2013; accession number, KF602048.1), all from linear plasmids. The chromosomal telomere of S. griseus 13350 [13] remains the sole member of the Sg13350 class, as do the telomeres of pRL1 and pRL2 of their eponymous classes [12].

Folding analysis of the six classes of telomeres indicated that all could form plausible stem-loop structures (Fig. 1 and individual Mfold projections in Fig. S4). Although there is little sequence similarity in the stems, the hairpins of the stem-loop structures across all six classes of telomeres are usually 3 or $4 \mathrm{nt}$ in length, with GAA, GGA and GTA the most common hairpin. With the exception of Tap/Tpg [10] and Tac/Tpc [15], which prime end-patching of archetypal and SCP1type telomeres, respectively, no other telomeric proteins have been characterized. Although a putative Tap was described in S. albidoflavus J1074 [41], we were unable to detect any similarity to Tap, Tpg, Tac or Tpc in genome sequences with Sg2247-type telomeres. This was also true in genomes with the Sg13350 and pLR1 types of telomeres, although pRL2 [12] encodes two candidate telomeric proteins: one displaying similarity to Tap and a helicase of Thiobacillus sp., whilst the other protein resembles Tpg and part of the adenovirus telomere terminal protein [12]. Taken together, this indicates that although streptomycete telomeres of all types bear some resemblance to each other in terms of the capacity of their $3^{\prime}$ overhangs to fold back on themselves, there are likely to be undiscovered systems able to catalyse end-patching.

We were only able to identify relatively few complete streptomycete genome sequences where all linear replicons were flanked by a member of these six telomere classes. Indeed, based on our strict criteria (complete sequence of all replicons and all replicons flanked by known telomeres), we could only confirm 20 closed genome sequences from streptomycetes. Many complete genomes within the NCBI database do not encode Tap and Tpg, so it is possible that other classes of telomeres await discovery. In a small-scale study using PCR, 8 out of 17 newly detected streptomycete linear plasmids lacked typical tap and tpg sequences and archetypal telomeres [12], but contained two undiscovered telomere types (pRL1-type and pRL2-type; Fig. 1). This suggests that novel streptomycete telomeres are common. If our analysis of replicon ends is representative of those of the entire family, it suggests that archetypal telomeres are the most common and phylogenetically widespread as they are found in two genera (Streptomyces and Kitasatospora) of the family Streptomycetaceae. In addition, it seems that Sg2247-types are less abundant and form a minority of the ends of both chromosomes and plasmids, whilst the other classes of telomeres are likely to be rare.

\section{Streptomycete telomeres are not distributed according to the evolutionary relatedness of different strains}

To determine whether the six different classes of streptomycete telomeres were associated with different phylogenetic clades within the family Streptomycetaceae, we first compiled a list of closed streptomycete sequences. As defined above, a closed genome sequence was that all replicons of that organism must be completely sequenced and that each linear replicon was flanked at both ends by one of the six classes of telomeres described previously. At the time of analysis (April 2020), there were 223 streptomycete genomes that were listed as complete in the NCBI database (1 from the genus Streptacidiphilus, 4 from Kitasatospora and 218 from Streptomyces). After inspecting these genomes for the presence of telomeres located at both ends of all linear replicons, we identified 20 strains that met these criteria (Table S3). The replicons of other genome sequences recorded as complete in the NCBI database might be flanked by undiscovered telomeres. As the $5^{\prime}$ ends of streptomycete replicons are covalently bound to a telomeric protein, sequencing approaches, such as Illumina, that employ paired-end reads may struggle to sequence to the very ends of linear replicons. In addition, the presence of TIRs flanking linear replicons may create assembly challenges using software tools designed for circular replicons. Taken together, it is unsurprising that many streptomycete genome sequences are not closed. We next organized all linear replicons carried by these 20 strains so that they were placed 


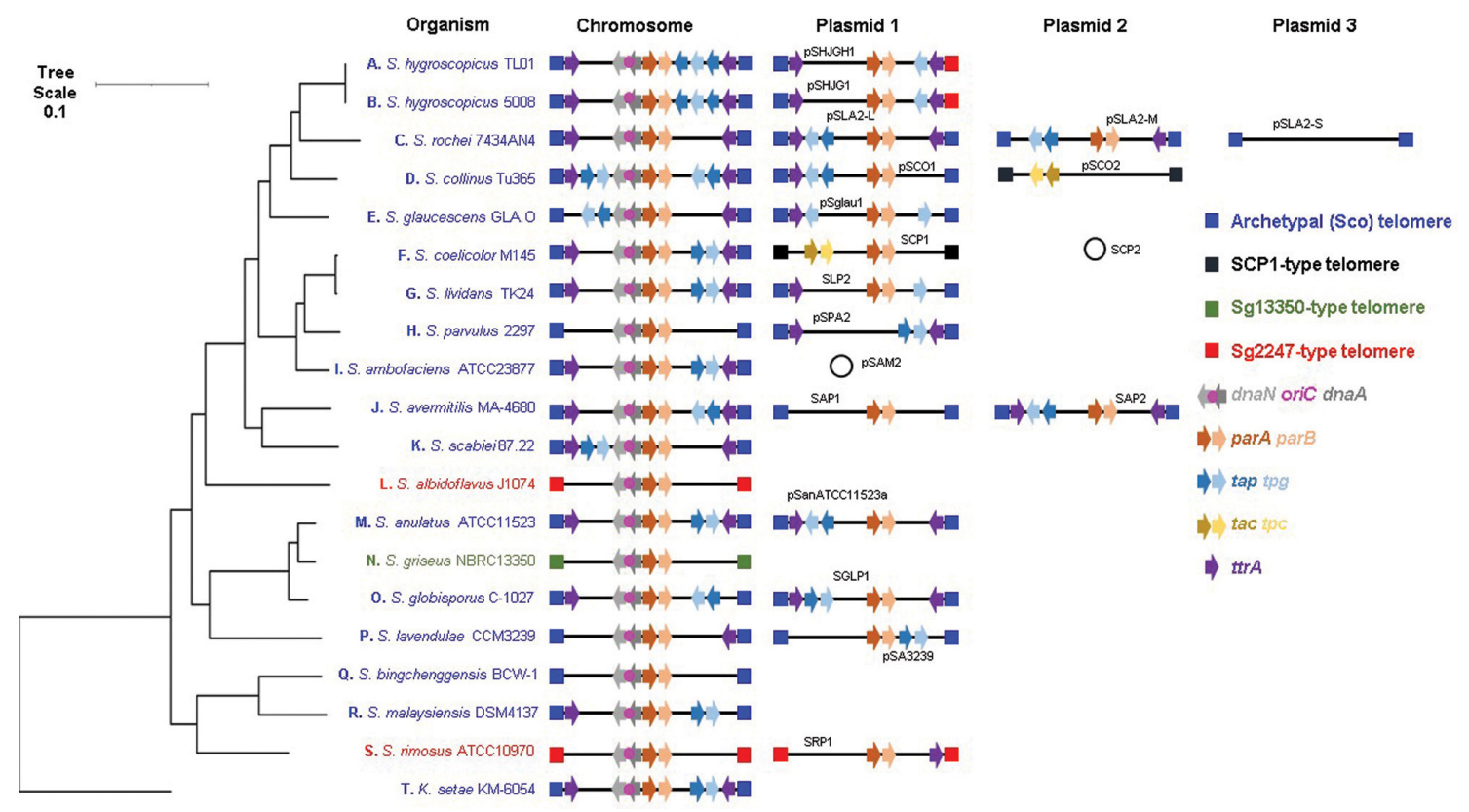

Fig. 3. Relative positions of genes encoding telomeric and partitioning proteins with respect to oriC and telomere class in the family Streptomycetaceae. All replicons carried by the 20 organisms with closed genomes (both chromosomes and plasmids) were investigated for the presence of genes encoding the archetypal and non-archetypal telomeric proteins, Tap/Tpg and Tac/Tpc, respectively. In addition, genes encoding TtrA, DnaN, DnaA, ParA and ParB are plotted (Table S3). Four of the six different classes of streptomycete telomeres are also displayed (archetypal (Sco), blue; SCP1, black; Sg2247, red; Sg13350, green). The locations of these genes and telomeres were plotted in relation to the whole genome MLSA described in Fig. S5. Branch lengths represent the evolutionary time between two nodes (units: substitutions per sequence site).

in the same direction based on the oriC region of $S$. coelicolor $[25,44]$ (Fig. 3). Replicons were orientated so that dnaN and dnaA were transcribed with the bottom strand as the coding strand, and parA and parB transcribed with the top strand as the coding strand. Chromosomes were organized in this orientation to comply with the established orientation for $S$. coelicolor M145, which has SCO0001 on the left and SCO7846 on the right [25]. Subsequently, we organized the 20 closed strains phylogenetically using OrthoANI [30] and AutoMLST [29] with $K$. setae KM-6054 as an outgroup by generating a maximum-likelihood tree and determining the location of strains with different classes of telomeres on this tree (Fig. S5). The high-resolution species tree shows that although archetypal telomeres are the most common of the chromosome ends, two phylogenetically separated strains (S. albidoflavus J1074 and S. rimosus ATCC 10970) carry chromosomes with Sg2247-type telomeres and suggests that telomeric exchange through horizontal gene transfer has occurred. The telomeres of $S$. griseus NBRC 13350 were confirmed by physical recovery [13] and, at the time of writing, no other telomeres with similarity to the ends of this strain have been identified. Despite this, the closely related strains Streptomyces anulatus ATCC 11523 and Streptomyces globisporus C-1027 (Fig. S5) possess archetypal telomeres that also indicates that horizontal transfer of telomeres has taken place. Similarly, S. rimosus ATCC 10970 (Sg2247), Streptomyces malaysiensis DSM4137 (archetypal) and 'Streptomyces bingchenggensis'
BCW-1 (archetypal) are also phylogenetically related but possess telomeres from different classes. The only closed streptomycete genome from outside the genus Streptomyces is from K. setae KM-6054 with archetypal telomeres; there are no characterized telomeric sequences available for Streptacidiphilus. Both S. coelicolor M145 and Streptomyces collinus Tu 365 possess chromosomes with archetypal telomeres, but plasmids with SCP1-type telomeres (Fig. 3). Intriguingly, the two closely related strains of $S$. hygroscopicus subsp. jinggangensis [43] possess a chromosome with archetypal telomeres, but a hybrid GLP with one archetypal and one Sg2247-type telomere. Hybrid replicons are not unknown in streptomycetes as demonstrated by the construction of S. coelicolor 2106 that carries a $1.85 \mathrm{Mb}$ GLP (derived from SCP1), in addition to a $7.2 \mathrm{Mb}$ linear hybrid chromosome with one archetypal end from the chromosome and one SCP1-type end from the GLP and, thus, this strain possesses replicons with both archetypal and non-archetypal telomeres [45].

We used this collection of closed genomes to investigate the TIRs by alignment of both ends of all replicons. The 20 chromosomes had a mean size of 8846536.25 bp (range $6841649-11936683 \mathrm{bp}$ ) and were flanked with 37990 (range 13-237 $155 \mathrm{bp}$ ) perfect inverted repeats and 77092 (range 138-631 $365 \mathrm{bp}$ ) imperfect inverted repeats, whilst the 16 GLPs spread over 12 strains were on average 188460 bp in size (range 17 526-617 085 bp) with TIRs with 5775 bp mean 
perfect inverted repeats and $6552 \mathrm{bp}$ mean imperfect inverted repeats. On average, a perfect TIR constituted $0.34 \%(0.87 \%$ imperfect) of a streptomycete chromosome and 3.06\% (3.48\% imperfect) of a GLP.

Whilst all chromosomes encode dnaN, dnaA, parA and parB, not all plasmids carry parAB (pSLA2-S, Streptomyces rochei; $\mathrm{pSCO} 2$, S. collinus; and pSPA2, Streptomyces parvulus); presumably these plasmids do not require these proteins for partitioning, or their functions are provided in trans by the chromosomal copies of these genes. We also mapped genes encoding known telomeric proteins ( $t a p / t p g$, tac/tpc and ttrA) (Fig. 3). SCP1-type telomeres flank two replicons, the GLPs SCP1 from S. coelicolor and pSSCO2 from S. collinus. Neither of these replicons encode Tap, Tpg nor $\operatorname{Ttr} A$, whilst both carry tac and $t p c$, encoding known non-archetypal telomeric proteins [15]. Consistent with previous analysis of S. albidoflavus J1074 [41], we were unable to locate either tap or tpg in the genomes of the two strains with Sg2247-type telomeres (S. albidoflavus J1074 or S. rimosus ATCC 10970) or S. griseus 13350 [13]. This suggests that their end-patching is carried out by a different mechanism to that of archetypal or SCP1-type telomeres and the identity of the proteins that catalyse this process remains elusive at the present time. The two closely related strains of S. hygroscopicus subsp. jinggangensis [43] contain plasmids with one archetypal end and one Sg2247-type telomere. These two strains carry two copies of tap on the chromosome and a copy of tpg on both the chromosome and plasmid. All strains with archetypal telomeres encode Tap and Tpg on at least one replicon, with the exception of 'S. bingchenggensis' BCW-1 [46]; it is unclear how this strain primes replication at the chromosome ends. Some strains (S. rochei 7434AN4 [47, 48], S. parvulus 2297, Streptomyces lavendulae CCM3239) do not carry tap and tpg on the chromosome, but do so on at least one GLP, suggesting that these plasmids are required to encode the telomeric proteins for chromosomal end-patching. Conversely, several GLPs do not encode telomeric proteins: SLP2 from 'Streptomyces lividans' (Tap), pSglaul from Streptomyces glaucescens GLA.0 (Tap), pSLA2-S from S. rochei 7434AN4 (Tap and Tpg) and SAP1 from Streptomyces avermitilis (Tap and Tpg), and suggest that end-patching is carried out by proteins encoded in trans or by other unknown proteins. Nevertheless, it seems that there is some crosstalk of the telomeric proteins and telomeres of different replicons contained within an individual strain.

Most replicons are flanked by inwardly transcribed copies of $t \operatorname{tr} A$, a putative DEAD-box helicase that is required for conjugation of the GLP, SLP2 in 'S. lividans' [40]. When present, $\operatorname{tr} A$ is often found towards the ends of replicons that are flanked by archetypal telomeres and is transcribed in an inward direction, suggesting that its orientation with respect to the telomeres is important for its function or transcriptional regulation. Exceptions to this are the S. parvulus 2297 and 'S. bingchenggensis' BCW-1 chromosomes, although the former strain carries a plasmid (pSPA2) that perhaps provides TtrA functionality in trans.
This gene is less common at the ends of replicons with non-archetypal, Sg13350- or the Sg2247-type replicon of S. albidoflavus J1074. Despite this, S. rimosus encodes a copy of $t$ trA at each chromosome termini and one copy at the right end of SRP1; unusually, the latter gene is transcribed in an outward direction. Consequently, the role of TtrA in plasmid-mediated conjugation and presence of $t \operatorname{tr} A$ at both ends of most streptomycete replicons suggests that telomere exchange may have arisen through recombination of recipient chromosomes with incoming GLPs. The circular genetic maps of streptomycetes provides circumstantial evidence for association between chromosome ends and is supported by fluorescence in situ hybridization studies in $S$. coelicolor [49]. The telomeric proteins display both an intramolecular (Tpg-Tpg) and an intermolecular (Tpg-Tpc) physical association between different telomeric proteins that was demonstrated by chemical cross-linking [46]. This presents a problem for replicon segregation through the formation of replicon pseudo-dimers brought about by the in trans association of the telomeric proteins. In a mechanism first proposed in 2011, these pseudo-dimers can only be resolved through disassociation and association of the telomeric proteins with those of the other daughter replicon or the occurrence of recombination between the two replicons in order to resolve the pseudo-dimer [46]. The appearance of replicons with different telomeres reported here (Fig. 3) might then be brought about by resolution of pseudo-dimers by recombination. Of course, exchange of different telomeres might also occur by classical transposition or recombination alone [50], but it is difficult to see how pseudo-dimers might be resolved without telomeric protein exchange or recombination between the two replicons [46].

Genome plasticity and evolution of streptomycetes is generated through intrinsic spontaneous recombination in addition to horizontal gene transfer in the environment and actioned by non-homologous end joining and integration of genetic material at the healing site [51]. Following a recent investigation of natural streptomycetes, it was revealed that closely related strains showed a large diversity of TIRs that reflected telomere exchanges between the chromosome and GLPs, and suggests that streptomycete telomeres display extensive allelic exchange brought about by horizontal gene transfer [52]. Strains that have undergone chromosomal changes produce diversified secondary metabolites and secrete more antibiotics [53], so perhaps it is the capacity for genetic exchange brought about by linear replicons that contributes to the metabolic diversity and bioactivity of this bacterial group.

Consequently, our analysis of closed streptomycete genome sequences demonstrates telomeric incongruence with a whole-genome-based phylogeny of these strains. This fact, taken together with the consistency of the location of tra at both chromosomal and plasmids ends, coupled with the ability of GLPs to form hybrid ends (S. hygroscopicus subsp. jinggangensis), suggest that telomeric exchange between streptomycete replicons is a common occurrence. 


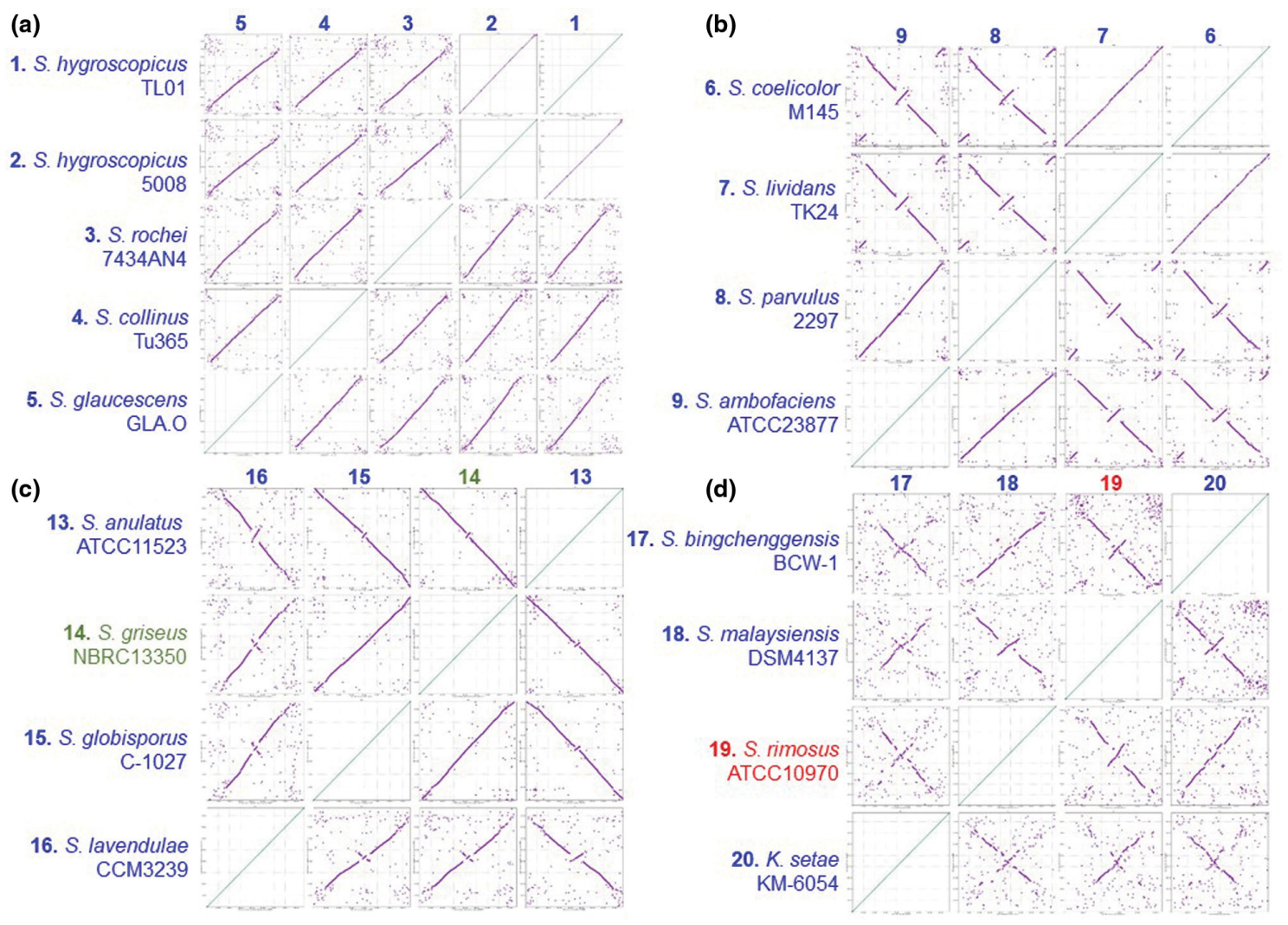

Fig. 4. Related strains display both syntenous and asynetenous chromosomes. Each of the 20 closed streptomycete chromosomes, organized so that the oriC regions were syntenous, were submitted to Nucmer and dot plots generated [32]. A comparison of all sequences is displayed in Fig. S7. Dot plots of strains around selected phylogenetic nodes (Fig. S5) are displayed here. (a) 1, S. hygroscopicus subsp. jinggangensis TL01; 2, S. hygroscopicus subsp. jinggangensis 5008; 3, S. rochei 7434AN4; 4, S. collinus Tu 365; 5, S. glaucescens GLA.0. (b) 6, S. coelicolor M145; 7, 'S. lividans' TK24; 8, S. parvulus 2297; 9, S. ambofaciens ATCC 23877. (c) 13, S. anulatus ATCC 11523; 14, S. griseus subsp. griseus NBRC 13350; 15, S. globisporus C-1027; 16, S. lavendulae subsp. lavendulae CCM3239. (d) 17, 'S. bingchenggensis' BCW-1; 18, S. malaysiensis DSM4137; 19, S. rimosus ATCC 10970; 20, K. setae KM-6054.

\section{A conserved origin island lies at the centre of streptomycete chromosomes}

When the 20 closed streptomycete chromosomes were orientated with $d n a A$ and $d n a N$ transcribed in a right to left direction, we noticed that parA and parB were transcribed in the opposite direction (Fig. 3). This suggested that the region containing oriC possessed conserved synteny across the analysed sequences. For this reason, we decided to investigate the extent of this synteny by carrying out a progressive alignment of the 20 closed chromosomes using Mauve [31]. This analysis showed a $\sim 50 \mathrm{~kb}$ locally collinear block (LCB), containing ori $C$, that lay in the core region of all chromosomes (brown arrow, Fig. S6), whilst other LCBs (green and purple arrows, Fig. S6) in the flanking regions displayed bilateral symmetry around oriC. For example, the green and purple LCBs occur in a similar orientation flanking oriC in closely related strains. In general, the green and purple LCBs are located on opposite replichores, except for the related strains 'S. bingchenggensis' BCW-1 and S. malaysiensis DSM4137 where both the green and purple LCBs are located on the same replichore. This suggests that recombination cannot occur within this core (brown) LCB and that, at the core of streptomycete chromosomes, there is a conserved region of $\sim 50 \mathrm{~kb}$ that we have designated the origin island.

We next investigated chromosome synteny in more detail through the generation of dot plots using Nucmer [32], where the chromosomal synteny of all strains was compared with each other and organized according to the MLSA tree for the 20 closed chromosomes displayed in Fig. S5. Dot plots for all 20 chromosomes are shown in Fig. S7 and for selected phylogenetic nodes in Fig. 4 that best illustrated chromosome synteny between related strains. Comparison of dot plots between sequences from related strains showed synteny across the entire length of the chromosomes, such as with the S. hygroscopicus node (Fig. 4a). Synteny is reduced towards the telomeres in comparison with the core region of the chromosome and likely reflects the genetic compartmentalization of streptomycete chromosomes that is consistent with the demonstration of a link between chromosome folding and gene expression in Streptomyces ambofaciens [54]. However, comparison of strains located at other phylogenetic nodes 


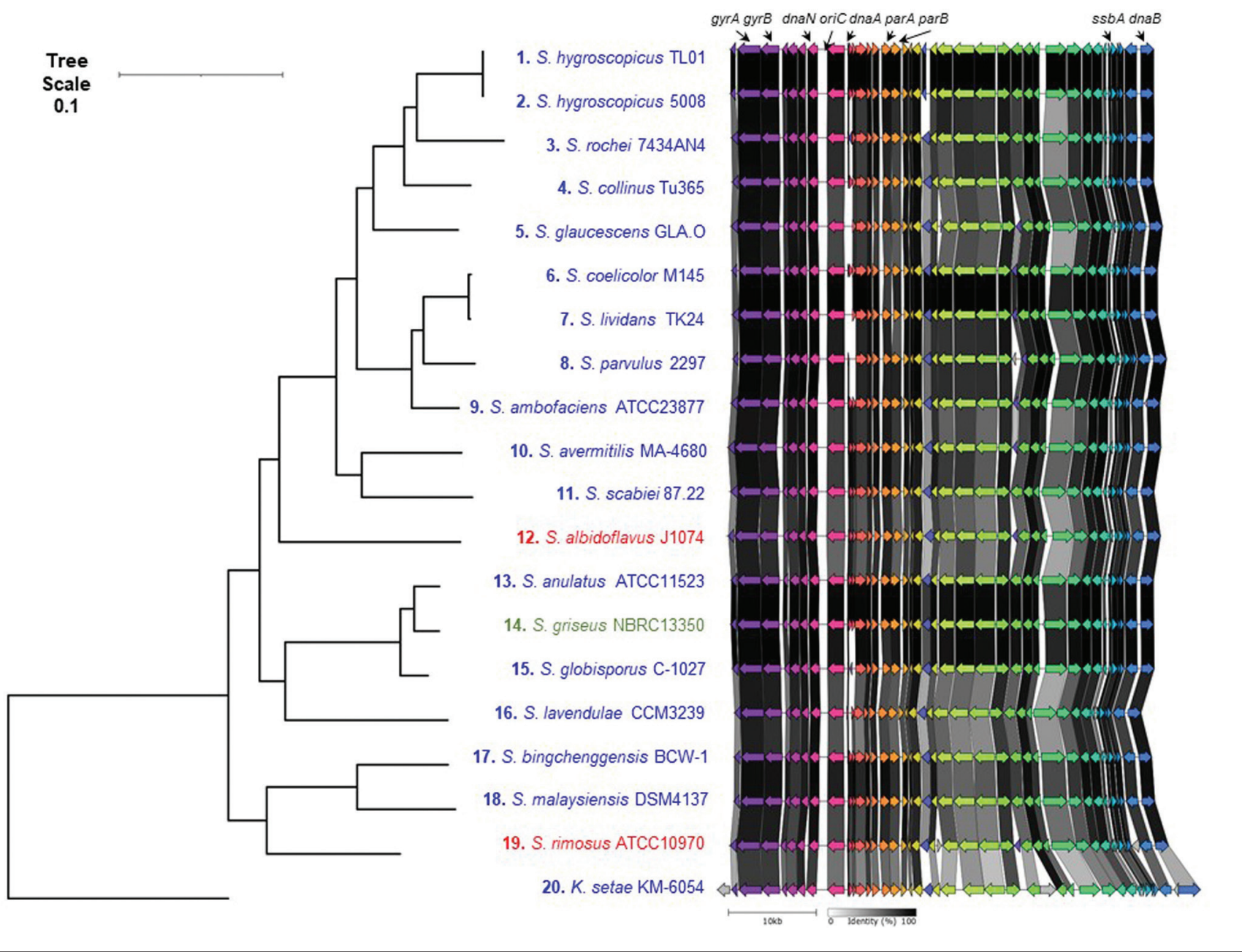

Fig. 5. Streptomycete origin islands are syntenous. Origin islands from tRNA-ile (left) and dnaB (right, SC03911) of 20 closed streptomycete genome sequences. Chromosomes with archetypal telomeres are listed in blue, Sg2247 telomeres in red and Sg13350 in green. Branch lengths represent the evolutionary time between two nodes (units: substitutions per sequence site).

(Fig. 4b-d) shows that one or more major chromosome inversions had taken place during the evolutionary divergence of $S$. coelicolor/'S. lividans' and S. parvulus/S. ambofaciens (Fig. 4b). Similar inversions were also identified at the S. griseus (Fig. 4c) and $S$. rimosus nodes (Fig. $4 \mathrm{~d}$ ). Interestingly, although the S. griseus 13350 chromosome carries the unique Sg13350 telomere class, its high average nucleotide identity with both S. anulatus and S. globisporus, which both possess archetypal telomeres (Fig. S5), suggests that telomere exchange was a relatively recent evolutionary event. The obvious bilateral symmetry between related chromosomes suggests that the recombination events leading to these rearrangements take place between the two replichores on either side of the origin island. It may be, therefore, that the conserved synteny of the origin island represents an irreducible axis around which recombination can take place, whilst not being subject to recombination itself. It also suggests that maintenance of an intact origin island is necessary for successful chromosome replication, segregation or appropriate compaction [54].
In $S$. coelicolor, this origin island contains the region corresponding to SCO3872-SCO3911 (Fig. 5) and includes gyrA, gyrB, recF, dnaN, oriC, dnaA, parA, parB, ssbA and dnaB. Across all 20 strains, the mean origin island size (Table S4) was $51055 \mathrm{bp}$ (range $48019-57671 \mathrm{bp}$ ) and constituted $0.59 \%$ of the chromosome (range $0.44-0.66 \%$ ), and its location was on average $48.49 \%$ (range $41.25-53.25 \%$ ) from the left-hand end of the replicon. Multiple genome alignments of streptomycete chromosomes show that they contain highly conserved core regions and variable sub-telomeric regions [55], and it has previously been reported that the central parts of streptomycete chromosomes are highly syntenic [56]. Whilst symmetrical rearrangements of streptomycete chromosomes have been reported before [41, 43], this is the first time, to our knowledge, that they have been related to a central axis. This suggests that the integrity of the origin island is important for maintenance of correct chromosome structure and function as when chromosome inversions take place, they do so in a symmetrical manner (between 


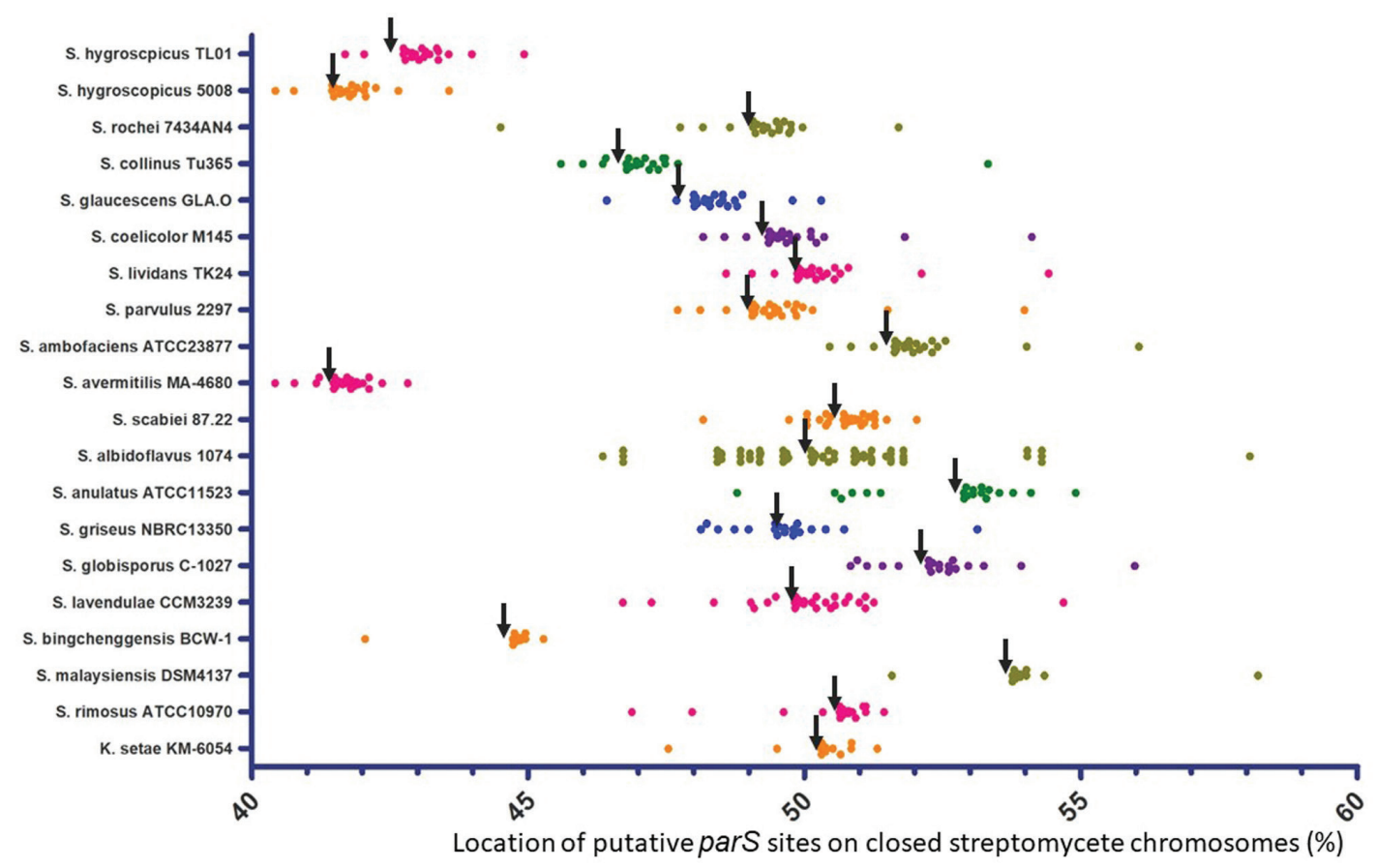

Fig. 6. Location of parS sites in the central $20 \%$ of streptomycete chromosomes. The locations of predicted parS sites on the chromosomes of the 20 closed streptomycete genomes were determined by interrogation of those genomes with the consensus matrix for bacterial parS sites [34]. Locations were expressed as a percentage of the chromosome size. The last base of dnaA from all genomes was selected as the location of oriC (black arrows).

replichores) rather than in an asymmetrical manner (within a replichore).

Bacterial chromosomes display a high degree of organization with respect to the locations of core genes [54, 57]. In Bordetella pertussis, with a circular chromosome, there is a bias toward symmetrical inversions [58] for the maintenance of replichore balance and preservation of favourable gene arrangements. Core-gene-defined genome organizational frameworks (cGOFs) can be classified as either symmetric or asymmetric with respect to their orientation in relation to the origin-terminus axis [59]; in Gram-positive organisms, cGOFs are exclusively symmetric and reversible in orientation [59]. As a result, it seems that the bilateral symmetry of streptomycete chromosomal rearrangements is consistent with Gram-positive bacteria with circular chromosomes and that a bias exists towards maintaining an approximate equality in the lengths of both replichores.

\section{Asymmetric distribution of parS sites in streptomycete chromosomes}

The delineation of the streptomycete origin island (Fig. 5) showed that this $\sim 50 \mathrm{~kb}$ region not only contained oriC between $d n a N$ and $d n a A$, but also a high number of putative parS sites located to the right of oriC. ParB binds to these sites and facilitates chromosome partitioning and connection with the polarisome $[20,21]$. As a result, we analysed the location of parS sites across the 20 closed streptomycete chromosomes. To do this, we employed a consensus matrix developed to locate putative prokaryotic parS sites [34].
The locations of these sites within the core region of the chromosome and with respect to oriC are shown in Fig. 6. The locations and sequences of these putative sites across all 20 closed genomes is provided in Supplementary File S1. On average, the number of parS sites per chromosome was 22.8 (range 11-65); most bacteria contain less than 5 parS sites [34], so the fact that streptomycetes carry up to 65 parS sites (S. albidoflavus J1074) [41] is remarkable. This strain, originally named Streptomyces albus J1074 [60], displays fast and dispersed growth in liquid culture [41]; it may be that the 65 predicted parS sites found in this strain contribute towards this through more efficient chromosome segregation and branching. As in S. coelicolor [20], most parS sites are located close to oriC in all strains and the origin island is abundant with parS sites; on average $45.24 \%$ of parS sites are located within the origin island with a mean distance of 5602 bp between parS sites in the origin island and 1113078 bp between parS sites in the bulk chromosome. Significantly, there is also a bias in the location of parS sites towards the right-hand side of the chromosome (Fig. 6). A total of $20.14 \%$ of parS sites lie to the left of oriC and $79.86 \%$ to the right (Table S4), meaning that during DNA replication and partitioning it is likely that the right replichore carries a greater abundance of $\mathrm{ParB} /$ parS nucleoprotein complexes with potential implications for chromosome segregation and connection of the chromosome to the hyphal tip. We did not include Streptomyces venezuelae in our analysis as the telomeres of its chromosome have not yet been established [61]; although, the possession of tap and tpg suggests that they resemble the archetypal telomeres of $S$. coelicolor 
M145. However, ChIP-seq analysis confirmed the formation of large nucleoprotein ParB complexes located at 16 parS sites in S. venezuelae 10712 [62] and, recently, the role of ParB in promoting chromosome inter-arm contact was reported [63]. parS sites are also located to the right of oriC in the S. venezuelae chromosome (data not shown). The significance of this bias of parS sites on the right replichore is unclear; it is true that only one daughter chromosome is directed towards the hyphal tip by ParB in S. coelicolor [21], but it is difficult to explain how a bias in ParB binding to the right replichore would manifest itself in the preferential direction of one daughter chromosome toward the hyphal tip.

\section{Conclusion}

In summary, following the completion of the S. rimosus ATCC 10790 genome sequence, the parental strain of an important lineage of industrial bacteria, we first characterized an uncommon set of telomeres for this organism before comparing them to the other five classes of streptomycete replicon ends. Through the organization of the closed replicons of 20 streptomycete chromosomes with respect to their phylogeny and physical orientation, we determined that the telomeres were not associated with particular clades and were likely shared amongst different strains by horizontal gene transfer. We identified an origin island that forms an axis around which symmetrical chromosome inversions can take place and that there is a bias in parS sites to the right of oriC in closed streptomycete genomes. These results open up new questions for further investigations towards understanding the mechanisms of end-patching of streptomycete chromosomes and the biological meaning that lies in the parS site bias. As such, understanding the fundamental structure of genome organization and mechanisms will prove indispensable in strain engineering for improved specialized metabolite production by this industrially important microbial group.

\section{Funding information}

This work was supported by studentships awarded by the Industrial Biotechnology Innovation Centre (L.A.-G.) and the University of Strathclyde (D.R.M.), as well as a Tools and Resources Development Fund award from the Biotechnology and Biological Sciences Research Council (grant number BB/M018792/1) (J.K.S.) awarded to P.R.H.

\section{Author contributions}

L.A.-G. and J.K.S. carried out experimental work. L.A.-G., D.R.M. and P.R.H. carried out data visualization and data curation. P.R.H. wrote the manuscript, whilst all authors were involved with review and editing of the manuscript. I.S.H. provided the resources and P.R.H. provided the conceptualization for the study.

\section{Conflicts of interest}

The authors declare that there are no conflicts of interest

\section{References}

1. Algora-Gallardo L, Schniete J, Mark DR, Hunter IS, Herron PR. Bilateral symmetry of linear streptomycete chromosomes. Figshare 2021.

2. Wang XJ, Yan YJ, Zhang B, An J, Wang JJ, et al. Genome sequence of the milbemycin-producing bacterium Streptomyces bingchenggensis. J Bacteriol 2010;192:4526-4527.
3. van Bergeijk DA, Terlouw BR, Medema MH, van Wezel GP. Ecology and genomics of Actinobacteria: new concepts for natural product discovery. Nat Rev Microbiol 2020;18:546-558.

4. McCormick JR, Flardh K. Signals and regulators that govern Streptomyces development. FEMS Microbiol Rev 2012;36:206-231.

5. Trojanowski D, Holowka J, Zakrzewska-Czerwinska J. Where and when bacterial chromosome replication starts: a single cell perspective. Front Microbiol 2018;9:2819.

6. Lin YS, Kieser HM, Hopwood DA, Chen CW. The chromosomal DNA of Streptomyces lividans 66 is linear. Mol Microbiol 1993;10:923-933.

7. Ruckert C, Szczepanowski R, Albersmeier A, Goesmann A, Iftime D, et al. Complete genome sequence of the kirromycin producer Streptomyces collinus Tu 365 consisting of a linear chromosome and two linear plasmids. J Biotechnol 2013;168:739-740.

8. Huang $\mathrm{C}-\mathrm{H}$, Lin Y-S, Yang Y-L, Huang S, Chen CW. The telomeres of Streptomyces chromosomes contain conserved palindromic sequences with potential to form complex secondary structures. Mol Microbiol 1998;28:905-916.

9. Nouioui I, Carro L, Garcia-Lopez M, Meier-Kolthoff JP, Woyke T, et al. Genome-based taxonomic classification of the phylum Actinobacteria. Front Microbiol 2018:9:2007.

10. Yang CC, Tseng SM, Pan HY, Huang CH, Chen CW. Telomere associated primase Tap repairs truncated telomeres of Streptomyces. Nucleic Acids Res 2017:45:5838-5849.

11. Goshi K, Uchida T, Lezhava A, Yamasaki M, Hiratsu K, et al. Cloning and analysis of the telomere and terminal inverted repeat of the linear chromosome of Streptomyces griseus. J Bacteriol 2002;184:3411-3415

12. Zhang R, Yang $Y$, Fang $P$, Jiang $C, X u L$, et al. Diversity of telomere palindromic sequences and replication genes among Streptomyces linear plasmids. Appl Environ Microbiol 2006;72:5728-5733.

13. Ohnishi $Y$, Ishikawa J, Hara $H$, Suzuki H, Ikenoya M, et al. Genome sequence of the streptomycin-producing microorganism Streptomyces griseus IFO 13350. J Bacteriol 2008;190:4050-4060.

14. Bao K, Cohen SN. Recruitment of terminal protein to the ends of Streptomyces linear plasmids and chromosomes by a novel telomere-binding protein essential for linear DNA replication. Genes Dev 2003;17:774-785.

15. Huang $\mathrm{CH}$, Tsai HH, Tsay YG, Chien YN, Wang SL, et al. The telomere system of the Streptomyces linear plasmid SCP1 represents a novel class. Mol Microbiol 2007;63:1710-1718.

16. Petkovic H, Cullum J, Hranueli D, Hunter IS, Peric-Concha N, et al. Genetics of Streptomyces rimosus, the oxytetracycline producer. Microbiol Mol Biol Rev 2006;70:704-728.

17. Pethick FE, MacFadyen AC, Tang Z, Sangal V, Liu TT, et al. Draft genome sequence of the oxytetracycline-producing bacterium Streptomyces rimosus ATCC 10970. Genome Announc 2013:1:e0006313.

18. Gravius B, Glocker D, Pigac J, Pandža K, Hranueli D, et al. The $387 \mathrm{~kb}$ linear plasmid pPZG101 of Streptomyces rimosus and its interactions with the chromosome. Microbiology 1994:140:2271-2277.

19. Pandza K, Pfalzer G, Cullum J, Hranueli D. Physical mapping shows that the unstable oxytetracycline gene cluster of Streptomyces rimosus lies close to one end of the linear chromosome. Microbiology 1997;143:1493-1501.

20. Jakimowicz D, Chater K, Zakrzewska-Czerwinska J. The ParB protein of Streptomyces coelicolor A3(2) recognizes a cluster of pars sequences within the origin-proximal region of the linear chromosome. Mol Microbiol 2002;45:1365-1377.

21. Kois-Ostrowska A, Strzatka A, Lipietta N, Tilley E, ZakrzewskaCzerwińska J, et al. Unique function of the bacterial chromosome segregation machinery in apically growing Streptomyces - targeting the chromosome to new hyphal tubes and its anchorage at the tips. PLOS Genet 2016;12:e1006488.

22. Kieser T, Bibb MJ, Buttner MJ, Chater KF, Hopwood DA. Practical Streptomyces Genetics. Norwich: John Innes Foundation; 2000. 
23. Fan $Y$, Dai $Y$, Cheng $Q$, Zhang G, Zhang D, et al. A self-ligation method for PCR-sequencing the telomeres of Streptomyces and Mycobacterium linear replicons. J Microbiol Methods 2012;90:105-107.

24. Evans M, Dyson P. Pulsed-field gel electrophoresis of Streptomyces lividans DNA. Trends Genet 1993:9:72.

25. Bentley SD, Chater KF, Cerdeño-Tárraga AM, Challis GL, Thomson NR, et al. Complete genome sequence of the model actinomycete Streptomyces coelicolor A3(2). Nature 2002:417:141-147.

26. Tamura M, Lee K, Miller CA, Moore CJ, Shirako Y, et al. RNase E maintenance of proper FtsZ/FtsA ratio required for nonfilamentous growth of Escherichia coli cells but not for colony-forming ability. J Bacteriol 2006;188:5145-5152.

27. Kumar S, Stecher G, Li M, Knyaz C, Tamura K. MEGA X: molecular evolutionary genetics analysis across computing platforms. Mol Biol Evol 2018;35:1547-1549.

28. Zuker M. Mfold web server for nucleic acid folding and hybridization prediction. Nucleic Acids Res 2003:31:3406-3415.

29. Alanjary M, Steinke K, Ziemert N. AutoMLST: an automated web server for generating multi-locus species trees highlighting natural product potential. Nucleic Acids Res 2019;47:W276-W282.

30. Lee I, Ouk Kim Y, Park SC, Chun J. OrthoANI: an improved algorithm and software for calculating average nucleotide identity. Int J Syst Evol Microbiol 2016;66:1100-1103.

31. Darling AE, Mau B, Perna NT. ProgressiveMauve: multiple genome alignment with gene gain, loss and rearrangement. PloS One 2010;5:e11147.

32. Marcais G, Delcher AL, Phillippy AM, Coston R, Salzberg SL, et al. MUMmer 4: a fast and versatile genome alignment system. PLoS Comput Biol 2018;14:e1005944

33. Gilchrist CLM, Chooi Y-H. Clinker \& clustermap.js: automatic generation of gene cluster comparison figures. Bioinformatics 2021:37:2473-2475.

34. Livny J, Yamaichi Y, Waldor MK. Distribution of centromere-like parS sites in bacteria: insights from comparative genomics. J Bacteriol 2007:189:8693-8703.

35. Crooks GE, Hon G, Chandonia J-M, Brenner SE. WebLogo: a sequence logo generator. Genome Res 2004;14:1188-1190.

36. Turatsinze J-V, Thomas-Chollier M, Defrance M, van Helden J. Using RSAT to scan genome sequences for transcription factor binding sites and cis-regulatory modules. Nat Protoc 2008;3:1578-1588.

37. Park CJ, Andam CP. Within-species genomic variation and variable patterns of recombination in the tetracycline producer Streptomyces rimosus. Front Microbiol 2019;10:552.

38. Kinashi H. Giant linear plasmids in Streptomyces: a treasure trove of antibiotic biosynthetic clusters. J Antibiot 2011;64:19-25.

39. Finlay AC, Hobby GL, P'an SY, Regna PP, Routien JB, et al. Terramycin, a new antibiotic. Science 1950;111:85.

40. Huang $\mathrm{CH}$, Chen $\mathrm{CY}$, Tsai HH, Chen C, Lin YS, et al. Linear plasmid SLP2 of Streptomyces lividans is a composite replicon. Mol Microbiol 2003:47:1563-1576.

41. Zaburannyi N, Rabyk M, Ostash B, Fedorenko V, Luzhetskyy A. Insights into naturally minimised Streptomyces albus J1074 genome. BMC Genomics 2014;15:97.

42. Barbe V, Bouzon M, Mangenot S, Badet B, Poulain J, et al. Complete genome sequence of Streptomyces cattleya NRRL 8057, a producer of antibiotics and fluorometabolites. J Bacteriol 2011;193:5055-5056.

43. Wu H, Qu S, Lu C, Zheng H, Zhou X, et al. Genomic and transcriptomic insights into the thermo-regulated biosynthesis of validamycin in Streptomyces hygroscopicus 5008. BMC Genomics 2012;13:337.

44. Huang TW, Hsu CC, Yang HY, Chen CW. Topoisomerase IV is required for partitioning of circular chromosomes but not linear chromosomes in Streptomyces. Nucleic Acids Res 2013;41:10403-10413.
45. Yamasaki M, Kinashi $H$. Two chimeric chromosomes of Streptomyces coelicolor A3(2) generated by single crossover of the wild-type chromosome and linear plasmid SCP1. J Bacteriol 2004:186:6553-6559

46. Tsai HH, Huang CH, Tessmer I, Erie DA, Chen CW. Linear Streptomyces plasmids form superhelical circles through interactions between their terminal proteins. Nucleic Acids Res 2011:39:2165-2174.

47. Nindita Y, Cao Z, Fauzi AA, Teshima A, Misaki Y, et al. The genome sequence of Streptomyces rochei 7434AN4, which carries a linear chromosome and three characteristic linear plasmids. Sci Rep 2019:9:10973.

48. Nindita Y, Cao Z, Yang Y, Arakawa K, Shiwa Y, et al. The tap-tpg gene pair on the linear plasmid functions to maintain a linear topology of the chromosome in Streptomyces rochei. Mol Microbiol 2015;95:846-858

49. Yang MC, Losick R. Cytological evidence for association of the ends of the linear chromosome in Streptomyces coelicolor. J Bacteriol 2001:183:5180-5186

50. Chen $\mathrm{CW}$, Huang $\mathrm{C}-\mathrm{H}$, Lee $\mathrm{H}-\mathrm{H}$, Tsai $\mathrm{H}-\mathrm{H}$, Kirby R. Once the circle has been broken: dynamics and evolution of Streptomyces chromosomes. Trends Genet 2002;18:522-529.

51. Hoff G, Bertrand C, Piotrowski E, Thibessard A, Leblond P. Genome plasticity is governed by double strand break DNA repair in Streptomyces. Sci Rep 2018:8:5272.

52. Tidjani AR, Bontemps C, Leblond P. Telomeric and sub-telomeric regions undergo rapid turnover within a Streptomyces population. Sci Rep 2020;10:7720.

53. Zhang Z, Du C, de Barsy F, Liem M, Liakopoulos A, et al. Antibiotic production in Streptomyces is organized by a division of labor through terminal genomic differentiation. Sci Adv 2020;6:eaay5781.

54. Lioy VS, Lorenzi J-N, Najah S, Poinsignon T, Leh H, et al. Dynamics of the compartmentalized Streptomyces chromosome during metabolic differentiation. Nature 2021:12:5221.

55. Bu Q-T, Li Y-P, Xie H, Wang J, Li Z-Y, et al. Comprehensive dissection of dispensable genomic regions in Streptomyces based on comparative analysis approach. Microb Cell Fact 2020;19:99.

56. Choulet F, Aigle B, Gallois A, Mangenot S, Gerbaud C, et al. Evolution of the terminal regions of the Streptomyces linear chromosome. Mol Biol Evol 2006;23:2361-2369.

57. Slager J, Veening JW. Hard-wired control of bacterial processes by chromosomal gene location. Trends Microbiol 2016;24:788-800.

58. Weigand MR, Peng Y, Batra D, Burroughs M, Davis JK, et al. Conserved patterns of symmetric inversion in the genome evolution of Bordetella respiratory pathogens. mSystems 2019;4:e00702-19.

59. Kang Y, Gu C, Yuan L, Wang Y, Zhu Y, et al. Flexibility and symmetry of prokaryotic genome rearrangement reveal lineage-associated core-gene-defined genome organizational frameworks. mBio 2014;5:e01867.

60. Labeda DP, Doroghazi JR, Ju K-S, Metcalf WW. Taxonomic evaluation of Streptomyces albus and related species using multilocus sequence analysis and proposals to emend the description of Streptomyces albus and describe Streptomyces pathocidini sp. nov. Int J Syst Evol Microbiol 2014;64:894-900.

61. Gomez-Escribano JP, Holmes NA, Schlimpert S, Bibb MJ, Chandra G et al. Streptomyces venezuelae NRRL B-65442: genome sequence of a model strain used to study morphological differentiation in filamentous actinobacteria. J Ind Microbiol Biotechnol 2021:kuab035.

62. Donczew M, Mackiewicz P, Wrobel A, Flardh K, ZakrzewskaCzerwinska J, et al. ParA and ParB coordinate chromosome segregation with cell elongation and division during Streptomyces sporulation. Open Biol 2016;6:150263.

63. Szafran M, Małecki T, Strzałka A, Pawlikiewicz K, Dutawa J, et al. Spatial rearrangement of the Streptomyces venezuelae linear chromosome during sporogenic development. Nat Comm 2021;12:5222. 